\title{
First observation of strong OZI rule violation in $\pi N$ interactions
}

\author{
A. Ferrer \\ Departamento de Física Atómica Molecular y Nuclear and IFIC \\ University of Valencia - CSIC, E-46100 Burjassot, Valencia, Spain \\ A.A. Grigoryan \\ Theoretical Department, Yerevan Physics Institute \\ Br. Alikhanian st.2 AM-375036 Yerevan, Armenia \\ V.F. Perepelitsa \\ ITEP, B. Cheremushkinskaya 25 \\ RU-11759 Moscow, Russia \\ P. Sonderegger
}

CERN CH-1211 Geneve 23 Switzerland

\begin{abstract}
The data of the CERN WA56 experiment that triggered the fast proton produced in the $\pi^{+} p$ and $\pi^{-} p$ interactions at beam momenta $20 \mathrm{GeV} / \mathrm{c}$ and $12 \mathrm{GeV} / \mathrm{c}$, respectively, are used to analyse the final states $p_{f} \phi(\omega) \pi^{ \pm}$. A large excess (up to two orders of magnitude) of the $\phi / \omega$ cross sections ratio over the OZI prediction is observed.
\end{abstract}

Key words: OZI violation, baryon exchange, phi, omega. 


\section{Introduction}

The Okubo-Zweig-Iizuka quark-line-rule [1] states that in the absence of strange particles the production of an ideal $\phi$ (strange quarks only) is forbidden. If the $\phi$ is produced only through the $\omega-\phi$ mixing, then the following relation between the cross sections of $\phi$ and $\omega$ - meson production holds:

$$
R=\frac{\sigma(A+B \rightarrow X+\phi)}{\sigma(A+B \rightarrow X+\omega)} \approx 6 \cdot 10^{-3}
$$

In (1) the states $A, B$ and $X$ do not contain strange quarks and the numerical value is the prediction of Gell-Mann-Okubo quadratic mass formula (the following PDG [2] values for the masses of vector mesons are used: $M_{\rho}=770 \mathrm{MeV}, M_{\omega}=782 \mathrm{MeV}, M_{\phi}=1019 \mathrm{MeV}$, and $\left.M_{K^{*}}=892 \mathrm{MeV}\right)$.

A large deviation from the value (1) was observed recently at LEAR in the processes of of $\bar{n} p, \bar{p} n$ and $\bar{p} p$ annihilation into final states $\phi(\omega) X(X=\pi, \gamma),[3-6]$, as well as in $\bar{p} d$ annihilation into $\phi(\omega) n[7]^{1}$

On the other hand, there is no dramatical deviation from (1) in $\phi$ and $\omega$ production in $p p$ as well as $\pi N$ interactions in the kinematical region where $\phi$ and $\omega$ are fast (forward production). For a review of experiments see refs. [8-10].

In this paper we present an analysis of a new class of reactions, namely the three-body processes

$$
\begin{aligned}
& \pi^{+} p \rightarrow p_{f} \phi \pi_{s}^{+} \\
& \pi^{+} p \rightarrow p_{f} \omega \pi_{s}^{+}
\end{aligned}
$$

at beam momentum $20 \mathrm{GeV} / \mathrm{c}$ and

$$
\begin{aligned}
& \pi^{-} p \rightarrow p_{f} \phi \pi_{s}^{-}, \\
& \pi^{-} p \rightarrow p_{f} \omega \pi_{s}^{-}
\end{aligned}
$$

at $12 \mathrm{GeV} / \mathrm{c}$ in the kinematical configuration where $p_{f}$ is a fast flying proton (with lab momentum $\geq p_{\text {beam }} / 2$ ) and the pion is the slowest particle in the lab. frame. This configuration corresponds physically to the baryon exchange processes (so-called backward production). We use the data of WA56 experiment [11] carried out at CERN in the 1980's with the aim to look for baryonium in baryon exchange processes. A very large excess

\footnotetext{
${ }^{1}$ In ref. [8] Okubo quotes the unpublished result of Syracuse group,

$$
\frac{\sigma\left(\bar{p} n \rightarrow \pi^{-} \phi\right)}{\sigma\left(\bar{p} n \rightarrow \pi^{-} \omega\right)}=0.253 \pm 0.059
$$

for annihilation at rest, obtained in a 1966 bubble chamber experiment at BNL. This ratio is compatible with OBELIX result [5]
} 
(up to two orders in magnitude) over the value of (1) is observed for the ratio of $\phi$ and $\omega$ production cross sections in the processes (2)-(5).

\section{Brief description of the WA56 set-up}

The layout of the $\Omega$ spectrometer as used in the WA56 experiment is described in detail in $[11,12]$. We recall here that the main trigger requirement was the detection of a fast forward proton with momentum greater than $10(7) \mathrm{GeV} / \mathrm{c}$ in $\pi^{+} p\left(\pi^{-} p\right)$ reactions at the incident momentum 20 (12) $\mathrm{GeV} / c$. Other important trigger requirement was the vetoing of events containing antiprotons of momenta greater than $2.6 \mathrm{GeV} / \mathrm{c}$.

These trigger conditions were imposed in order to separate the kinematical region which corresponds to baryon exchange $\left(0 \geq u_{1} \geq-1.5 \mathrm{GeV}^{2}\right)$ in the crossed, $u_{1}$-channel (see Fig. 1) and to suppress a difractive background with fast antiprotons from processes like $\pi^{ \pm} p \rightarrow p_{f} \bar{p} \pi^{ \pm} p_{s}$.

To fulfill the identification of particles, we used a TOF system and $d E / d x$ information from the scintillation barrel counters surrounding the $\mathrm{H}_{2}$ target.

The nominal experimental sensitivities corresponding to the WA56 data presented here are 150 events/nb in the $\pi^{+} p$ exposure and 30 events/nb in the $\pi^{-} p$ one.

\section{Channel $\pi p \rightarrow p_{f} \phi \pi$.}

We analyze the reactions (2) and (4) using the data on the following processes with 4 particles in the final state.

$$
\pi^{ \pm} p \rightarrow p_{f} K^{+} K^{-} \pi_{s}^{ \pm}
$$

where the pion $\pi_{s}$ was the slowest particle in the final state. As a rule, such particles were not detected by the WA56 tracking apparatus. So, from the methodological point of view, the problem of identification of the processes (3) and (5) with the slow pion is similar to that of the work [12] where the study of the central production of the $\rho^{0}, \mathrm{f}_{2}, \rho_{3}^{0}$ mesons in the baryon exchange reaction

$$
\pi^{+} p \rightarrow p_{f} \pi^{+} \pi^{-} \pi_{s}^{+}
$$

was done. There, we used the sample of events of topology ++- that comes from the $4-$ prong events with one undetected slow particle. This particle was reconstructed imposing 1-C kinematical fit. In this work we follow the same procedure. First, the missing mass square $\left(M M^{2}\right)$ was calculated with $p, K^{+}, K^{-}$mass hypotheses for the detected particles, requiring the $K^{+}$and $K^{-}$mass assignments were compatible with the response of the particle identificators ( $\mathrm{C} 1$, TOF system, barrel $d E / d x$ measurements). The events were 
selected imposing the cuts $\left|M M^{2}-m_{\pi}^{2}\right|<0.12\left(\mathrm{GeV} / \mathrm{c}^{2}\right)^{2}$ (in the $\pi^{+} p$ exposure) and $\left|M M^{2}-m_{\pi}^{2}\right|<0.06\left(\mathrm{GeV} / \mathrm{c}^{2}\right)^{2}$ (in the $\pi^{-} p$ exposure). Then a $1-\mathrm{C}$ momentum-energy balance fit was done and only the events with a $P\left(\chi^{2}\right)>5 \%$ were retained.

The spectrum of $K^{+} K^{-}$mass of selected events of reaction (6) with $\pi^{+}$beam is shown in Fig. 2a. The signal of the $\phi(1020)$ meson is clearly seen in this spectrum, with signal to background ratio about 1.4. The main part of the background under the $\phi$ peak (as well as in the whole spectrum of Fig. 2a) was found to come from the $\pi^{+} \pi^{-}$pairs of the reaction (7). In particular, the wide bump around $1.25 \mathrm{GeV} / \mathrm{c}^{2}$ is a reflection of the $\rho^{0}$ meson produced in this reaction. Fig. $2 \mathrm{~b}$ shows the distribution of the selected events in which either $K^{+}$or $K^{-}$was proved (using the particle identificators) not to be a pion. It is seen that the signal to background ratio for the $\phi$ peak increases substantially under this selection reinforcing the validity of the channel identification. Further, to check directly that the peak at $\phi$ meson mass in Fig. 2a is not a reflection of some resonance or $\Lambda$ hyperon we have tried other reasonable mass assignments $\left(K^{+} \pi^{-}, \pi^{+} K^{-}, p \pi^{-}, \bar{p} \pi^{+}, K^{+} \bar{p}, p K^{-}\right)$. All these assignments converted the peak to broad structures that could not be identified with any well-established resonance (or $\Lambda$ hyperon) in the corresponding channel.

The curves in Fig. 2 are the mass fit results. The fit was done with a parameterization of the $K^{+} K^{-}$mass spectra of the form

$$
A\left(M-2 M_{0}\right)^{\alpha} \exp \left\{\left(2 M_{0}-M\right) \beta\right\}(1+B W)
$$

where $M_{0}=2 m_{K}$ and $B W$ means a Breit-Wigner term. The second Breit-Wigner term was added to this expression when fitting the mass spectrum in Fig. 2a in order to get a description of the reflection bump at $1.25 \mathrm{GeV} / \mathrm{c}^{2}$ mentioned above.

The $K^{+} K^{-}$mass spectrum of events fitting the reaction (6) with $\pi^{-}$beam is shown in Fig. 3. The $\phi$ meson signal is seen there, with a lower statistical significance of the peak, however, greater than 4 s.d.

We present the cross sections for the $\phi$ meson production in two ways. First, we deplete the full signal in Fig. $2 \mathrm{a}\left(\pi^{+} p\right.$ exposure) into five c.m.s. rapidity bins and that of $\pi^{-} p$ exposure (Fig. 3) into two rapidity bins. The number of events after background subtraction, the acceptances, and the calculated cross sections are displayed in Table 1 together with the integrated values for the full rapidity intervals presented. Note, the cross sections given are corrected for the $\phi \rightarrow K^{+} K^{-}$decay branching ratio (49\%).

Another presentation of the production cross sections, in terms of the $\phi \pi$ mass is given in Table 2.

\section{Channel $\pi p \rightarrow p_{f} \omega \pi$.}

We analyze the processes (3) and (5) using the following reactions with 5 particle in the final state 


$$
\pi^{ \pm} p \rightarrow p_{f} \pi^{+} \pi^{-} \pi^{0} \pi_{s}^{ \pm}
$$

where $\pi_{s}^{+}$(or $\pi_{s}^{-}$), not associated to the $\omega$, was the slowest particle in the final state, and it was not detected by $\Omega$ spectrometer chambers, similarly to the $\pi_{s}$ in reactions (6) and (7). Now the situation is more complicated since in addition to this pion, the $\pi^{0}$ from the $\omega$ decay is unseen. In total, we have 6 unknown kinematical variables (namely, the 3momenta components of $\pi_{s}$ and $\pi^{0}$ ) and 4 constraints (energy-momentum conservation). However, using information from the barrel $(d E / d x$ and azimuthal angle) the number of constraints can be enlarged up to 6 providing a $0-\mathrm{C}$ kinematical balance. To ensure its unambiguity we used only those ("clean barrel") events which have had a single fired barrel element non-associated with the detected tracks. More detailed description of the $0-\mathrm{C}$ fit procedure will be given in [15]. The reconstruction of both, $\pi_{s}$ and $\pi^{0}$ momenta with this procedure was found to be good enough, the mass resolution for the $\pi^{+} \pi^{-} \pi^{0}$ system in the $\omega$ region being close to $60 \mathrm{MeV} / \mathrm{c}^{2}$. However, the selectivity of the procedure is very low. In order to suppress the background in the region of the $\omega$ we have applied kinematical cuts requiring the momenta of charged pions from the $\omega$ decay to be below 2 $\mathrm{GeV} / \mathrm{c}$ and that of the $\pi^{0}$ below $1.5 \mathrm{GeV} / \mathrm{c}$. These cuts affect strongly the background, and rather weakly the signal of the $\omega$, especially at low $\omega$ rapidities, as it has been proved with the MC simulation. With these cuts, the $\omega$ signal appears to be seen in the $\pi^{+} \pi^{-} \pi^{0}$ mass distributions shown in Fig. $4 \mathrm{a}\left(\pi^{+} p\right.$ exposure) and Fig. $4 \mathrm{~b}\left(\pi^{-} p\right.$ exposure), with signal to background ratios about 0.5 and 0.3 , respectively.

We present the results on the $\omega$ production cross sections in the same manner as in the case of $\phi$. They are given in Tables 3 and 4 . Note, the $\omega$ signal in the last rapidity bin $(+0.5$ to +1$)$ was extracted omitting the cuts on $\pi$ momenta described above. Further, the efficiency decrease due to the selection of the "clean barrel" events is included in the acceptances given in Tables 3 and 4 . Again, the production cross sections given are corrected for the $\omega \rightarrow \pi^{+} \pi^{-} \pi^{0}$ decay branching ratio (89\%).

\section{The $\phi / \omega$ ratios.}

The ratios of the $\phi$ to $\omega$ production cross sections are displayed in Table 5 and Fig. 5 . They show a clear tendency to grow with the rapidity and the $\phi \pi(\omega \pi)$ invariant mass decrease. It is interesting to compare our ratios with those measured in $\bar{N} N$ annihilation at LEAR [4-6], since a direct physical correspondence between our processes and $\bar{N} N$ annihilation can exist. This is seen from the baryon exchange diagram represented in Fig. 1, where the systems $\phi \pi_{s}$ and $\omega \pi_{s}$ can be considered as if they are created in the annihilation of a virtual antibaryon $\left(\alpha_{\bar{B}}\right)$ on the on-mass-shell proton. In the case of $\pi^{-} p$ reaction, one deals with the "subannihilation" of virtual $\bar{\Delta}^{--}, \alpha_{\bar{\Delta}^{--}} p \rightarrow \phi(\omega) \pi_{s}^{-}$, while in $\pi^{+} p$ reaction both virtual antineutron and $\bar{\Delta}^{0}$ can, in principle, annihilate. The estimations show, however [15], that the contribution of $\Delta$ exchange into processes (2) and $(3)$ is expected to be negligible as compared to that of nucleon, and hence the systems 
$\phi \pi_{s}^{+}$and $\omega \pi_{s}^{+}$are produced in these processes via the subannihilation $\alpha_{\bar{n}} p$, an analog of $\bar{n} p$ on-mass-shell annihilation analyzed by OBELIX [4]. Recalling that the threshold of $N \bar{N}$ annihilation lies in our mass bin $1.75 \div 2.25 \mathrm{GeV} / \mathrm{c}^{2}$, one can see that our $\phi / \omega$ ratio exceeds those measured in $\bar{n} p$ [4], $\bar{p} n$ [5], and $\bar{p} p$ [6] annihilation. Together with this, a definite tendency of the $\phi / \omega$ ratio growth below the annihilation threshold $2 M_{N}$ is seen in our data.

\section{Conclusions}

We have identified the baryon exchange reactions $\pi^{ \pm} p \rightarrow p_{f} \phi \pi_{s}^{ \pm}, \pi^{ \pm} p \rightarrow p_{f} \omega \pi_{s}^{ \pm}$at 20 and $12 \mathrm{GeV} / \mathrm{c}$ using the WA56 experiment data obtained at the CERN $\Omega$ spectrometer.

We have then computed the $\phi, \omega$ production cross sections and obtained the $\phi / \omega$ ratio as a function of the $\phi, \omega$ rapidities and the $\phi \pi_{s}, \omega \pi_{s}$ invariant masses.

Our data show a strong OZI rule violation, by up to two orders in magnitude.

\section{Acknowledgements}

A.A.G. and V.F.P. thank the colleagues from IFIC for a warm hospitality and many useful discussions during their stay at Valencia University. The work done by A.A.G. was supported in part by the INTAS Grant No 93-0079 and by the Generalitat Valenciana visitor's Grant. 


\section{References}

[1] S. Okubo, Phys. Letters B 5 (1963) 165;

G. Zweig, CERN Report No.8419/TH412 (1964) unpublished;

I. Iizuka, Progr. Theor. Phys. Suppl. 37-38 (1966) 21

[2] Review of Particle Properties, Phys. Rev. D50 (1994) 1173

[3] J. Reifenróther et al., Phys. Lett. B 267 (1991) 299

[4] V.G. Ableev et al., Phys. Lett. B 334 (1994) 237

[5] V.G. Ableev et al., Nucl. Phys. A 585 (1995) 577

[6] C. Amsler et al., Phys. Lett. B 346 (1995) 363

[7] A Bertin et al., Yad. Fiz. 59 (1996) 1511 [ Phys. At. Nucl. (Engl. Transl.) 59 (1996) 1455 ]

[8] S. Okubo, Phys. Rev. D 16 (1977) 2336

[9] J. Ellis et al., Phys. Letters B 353 (1995) 319

[10] M.G. Sapozhnikov, JINR Preprint E15-95-544"Production of $\phi$-mesons in $\bar{N} N$ annihilation and polarized strangeness in the nucleon" Lecture at the XXIX St.Petersburg Winter School on Nuclear Physics and Elementary Particles, Zelenogorsk, 1995

[11] Z. Ajaltouni et al., Nucl. Phys. B 209 (1982) 301

[12] V.F. Perepelitsa et al., Z. Phys. C - Particles and Fields 52 (1991) 407

[13] A. Ferrer et al. To be published

[14] V.F. Perepelitsa et al., Z. Phys. C-Particles and Fields 50 (1991) 395

[15] A. Ferrer et al. First observation of strong OZI rule violation in $\pi N$ interactions proceeding via baryon exchange. In preparation. 


\section{Figure captions.}

Fig. 1 Baryon exchange diagram corresponding to the production of a fast proton plus a mesonic system $\phi(\omega) \pi_{s}$ in $\pi p$ collisions. $\alpha_{N}$ and $\alpha_{\Delta}$ are the virtual nucleon and $\Delta$, respectively.

Fig. 2) $K^{+} K^{-}$mass spectrum for the events of the $\pi^{+} p$ reaction which pass the $1-\mathrm{C}$ fit procedure and whose mass are assignments compatible with the particle identificator response: a) full spectrum;b) $K^{+}$or $K^{-}$are proved not to be a pion.

Fig. 3) The same as in Fig. 2a for $\pi^{-} p$ reaction.

Fig. 4) $\pi^{+} \pi^{-} \pi^{0}$ mass spectrum for events which pass the $0-\mathrm{C}$ fit procedure and are selected with the cuts $p_{\pi^{+}}, p_{\pi^{-}} \leq 2 \mathrm{GeV} / \mathrm{c}, p_{\pi^{0}} \leq 1.5 \mathrm{GeV} / \mathrm{c}$ : a) for $\pi^{+} p$ reaction; b) for $\pi^{-} p$ reaction.

Fig. 5) $\phi / \omega$ production cross section ratio as function of: a) c.m.s. rapidity of the $\phi(\omega)$ meson; b) the $\phi \pi(\omega \pi)$ mass. 
Table 1.

Number of events, acceptances and cross sections vs $\phi$ rapidity

\begin{tabular}{lccc}
\hline $\begin{array}{l}\text { Rapidity } \\
\text { interval }\end{array}$ & $\begin{array}{c}\text { Number of events } \\
\text { (background subtracted) }\end{array}$ & $\begin{array}{c}\text { Acceptance } \\
(\%)\end{array}$ & $\sigma$ (nb) \\
\hline $20 \mathrm{GeV} / \mathrm{c} \pi^{+} p$ data & $35 \pm 9$ & 2.4 & $19.8 \pm 5.3$ \\
\hline-1.5 to -1.0 & $88 \pm 13$ & 8.8 & $13.6 \pm 2.0$ \\
-1.0 to -0.5 & $49 \pm 10$ & 9.2 & $7.3 \pm 1.4$ \\
-0.5 to 0.0 & $47 \pm 9$ & 8.8 & $7.3 \pm 1.4$ \\
0.0 to 0.5 & $28 \pm 6$ & 6.2 & $6.1 \pm 1.4$ \\
0.5 to 1.0 & $247 \pm 22$ & & $54.1 \pm 6.2$ \\
Sum $(-1.5$ to 1.0$)$ & & & \\
\hline 12 GeV $/ \mathrm{c} \pi^{-} p$ data & $25 \pm 8$ & 9.4 & $18 \pm 6$ \\
\hline-0.5 to 0.0 & $19 \pm 6$ & 8.6 & $15 \pm 5$ \\
0.0 to 0.5 & $44 \pm 10$ & & $33 \pm 8$ \\
Sum $(-0.5$ to 0.5$)$ & &
\end{tabular}

Table 2.

Number of events, acceptances and cross sections vs $\phi \pi$ mass

\begin{tabular}{lccc}
\hline $\begin{array}{l}\text { Mass } \\
\text { interval, } \\
\mathrm{GeV} / \mathrm{c}^{2}\end{array}$ & $\begin{array}{c}\text { Number of events } \\
\text { (background subtracted) } \\
\text { or upper limit at } 95 \% \mathrm{CL}\end{array}$ & $\begin{array}{c}\text { Acceptance } \\
(\%)\end{array}$ & $\sigma(\mathrm{nb})$ \\
\hline $20 \mathrm{GeV} / \mathrm{c} \pi^{+} p$ data & $52 \pm 9$ & 4.4 & $16 \pm 3$ \\
\hline below 1.75 & $75 \pm 12$ & 8.6 & $12 \pm 2$ \\
1.75 to 2.25 & $53 \pm 10$ & 8.8 & $8.2 \pm 1.5$ \\
2.25 to 2.75 & $45 \pm 9$ & 8.8 & $6.9 \pm 1.4$ \\
2.75 to 3.25 & $20 \pm 7$ & 4.6 & $5.9 \pm 2.2$ \\
over 3.25 & & & \\
\hline $12 \mathrm{GeV} / \mathrm{c} \pi^{-} p$ data & $\leq 13$ & 6.7 & $\leq 13$ \\
\hline 1.75 to 2.25 & $16 \pm 5$ & 8.0 & $14 \pm 4$ \\
2.25 to 2.75 & $15 \pm 5$ & 6.8 & $15 \pm 5$ \\
2.75 to 3.75 & & & \\
\hline
\end{tabular}


Table 3.

Number of events, acceptances and cross sections vs $\omega$ rapidity

\begin{tabular}{lccc}
\hline $\begin{array}{l}\text { Rapidity } \\
\text { interval }\end{array}$ & $\begin{array}{c}\text { Number of events } \\
\text { (background subtracted) }\end{array}$ & $\begin{array}{c}\text { Acceptance } \\
(\%)\end{array}$ & $\sigma$ (nb) \\
\hline $20 \mathrm{GeV} / \mathrm{c} \pi^{+} p$ data & $12 \pm 4$ & & \\
\hline-1.5 to -1.0 & $69 \pm 14$ & 1.6 & $32.4 \pm 6.6$ \\
-1.0 to -0.5 & $162 \pm 22$ & 2.6 & $46.8 \pm 6.2$ \\
-0.5 to 0.0 & $62 \pm 13$ & 0.6 & $77.6 \pm 16.6$ \\
0.0 to 0.5 & $40 \pm 11$ & 0.6 & $50.1 \pm 13.8$ \\
0.5 to 1.0 & $345 \pm 32$ & & $237 \pm 26$ \\
Sum $(-1.5$ to 1.0$)$ & & & \\
\hline $12 \mathrm{GeV} / \mathrm{c} \pi^{-} p$ data & $32 \pm 8$ & 1.5 & $80 \pm 20$ \\
\hline-0.5 to 0.0 & $60 \pm 16$ & 2.0 & $113 \pm 30$ \\
0.0 to 0.5 & $92 \pm 18$ & & $193 \pm 36$ \\
Sum $(-0.5$ to 0.5$)$ & &
\end{tabular}

Table 4 .

Number of events, acceptances and cross sections vs $\omega \pi$ mass

\begin{tabular}{lccc}
\hline $\begin{array}{l}\text { Mass } \\
\text { interval, } \\
\mathrm{GeV} / \mathrm{c}^{2}\end{array}$ & $\begin{array}{c}\text { Number of events } \\
\text { (background subtracted) }\end{array}$ & $\begin{array}{c}\text { Acceptance } \\
(\%)\end{array}$ & $\sigma(\mathrm{nb})$ \\
\hline $20 \mathrm{GeV} / \mathrm{c} \pi^{+} p$ data & $45 \pm 12$ & 0.9 & $37 \pm 10$ \\
\hline below 1.75 & $131 \pm 20$ & 2.7 & $37 \pm 6$ \\
1.75 to 2.25 & $91 \pm 17$ & 1.4 & $49 \pm 9$ \\
2.25 to 2.75 & $37 \pm 15$ & 0.3 & $92 \pm 37$ \\
2.75 to 3.25 & $56 \pm 15$ & & \\
\hline $12 \mathrm{GeV} / \mathrm{c} \pi^{-} p$ data & $51 \pm 13$ & 2.0 & $105 \pm 28$ \\
\hline 1.75 to 2.25 & & 1.7 & $113 \pm 29$ \\
\hline 2.25 to 2.75 & &
\end{tabular}


Table 5.

Ratios of $\phi$ and $\omega$ production cross sections

\begin{tabular}{lccc}
\hline $\begin{array}{l}\text { Rapidity } \\
\text { interval }\end{array}$ & $\phi / \omega$ ratio & $\begin{array}{c}\phi \pi_{s}\left(\omega \pi_{s}\right) \text { mass } \\
\text { interval, } \mathrm{GeV} / \mathrm{c}^{2}\end{array}$ & $\phi / \omega$ ratio \\
\hline $20 \mathrm{GeV} / \mathrm{c} \pi^{+} p$ data & & & \\
\hline-1.5 to -1.0 & $0.66 \pm 0.28$ & below 1.75 & $0.43 \pm 0.14$ \\
-1.0 to -0.5 & $0.42 \pm 0.11$ & 1.75 to 2.25 & $0.32 \pm 0.08$ \\
-0.5 to 0.0 & $0.15 \pm 0.04$ & 2.25 to 2.75 & $0.17 \pm 0.04$ \\
0.0 to 0.5 & $0.09 \pm 0.03$ & 2.75 to 3.25 & $0.075 \pm 0.034$ \\
0.5 to 1.0 & $0.12 \pm 0.04$ & & \\
Sum $(-1.5$ to 1.0$)$ & $0.23 \pm 0.04$ & & \\
\hline $12 \mathrm{GeV} / \mathrm{c} \pi^{-} p$ data & & & $\leq 0.12$ \\
\hline-0.5 to 0.0 & $0.22 \pm 0.09$ & 1.75 to 2.25 & \\
0.0 to 0.5 & $0.13 \pm 0.06$ & 2.25 to 2.75 & $0.12 \pm 0.05$ \\
Sum $(-0.5$ to 0.5$)$ & $0.17 \pm 0.05$ & & \\
\hline
\end{tabular}




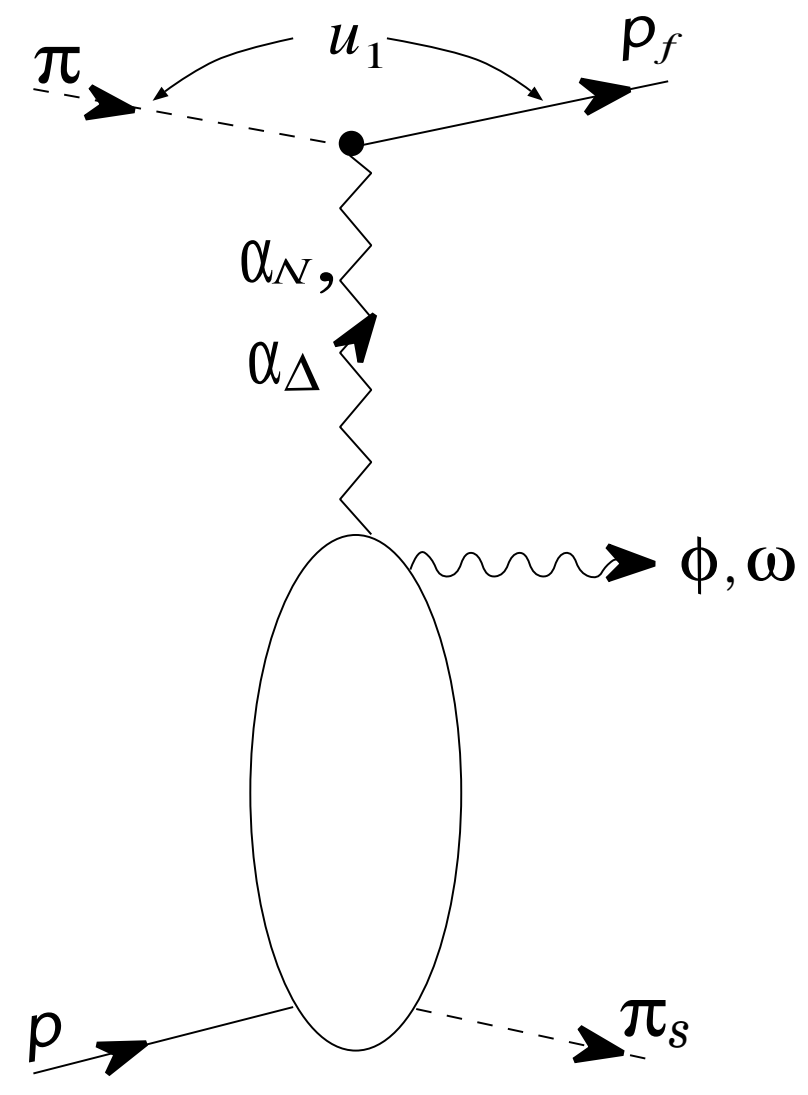

Fig. 1 


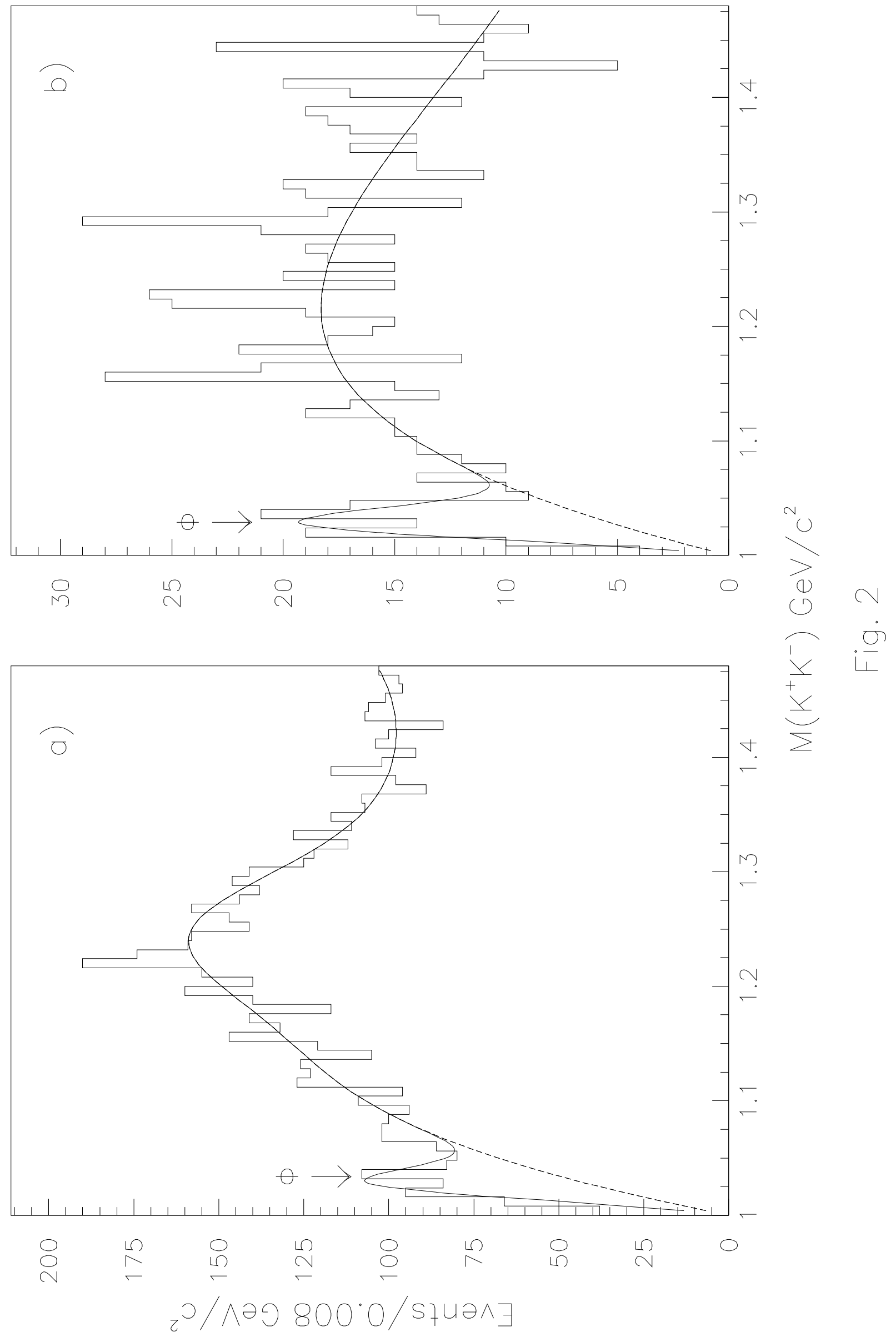




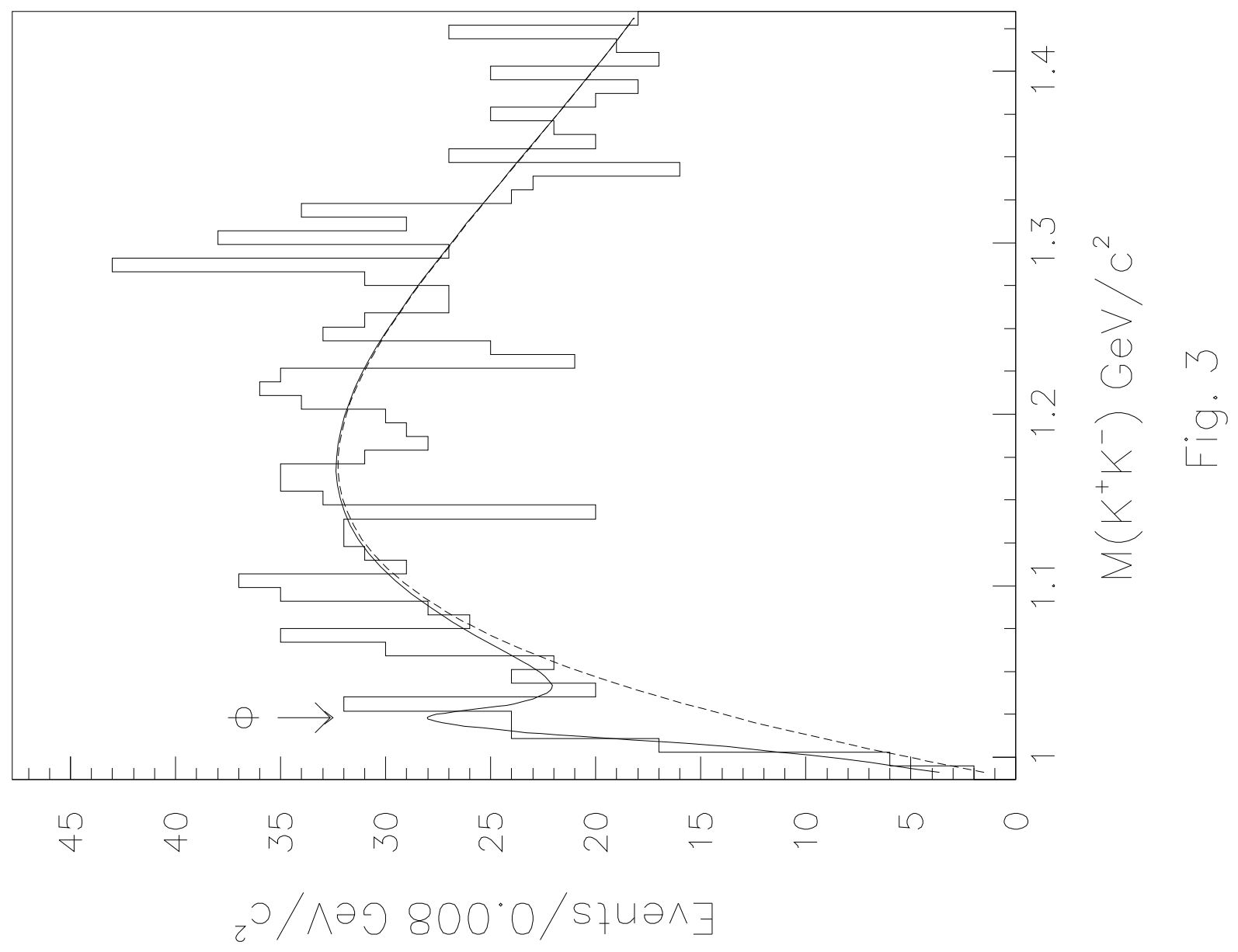




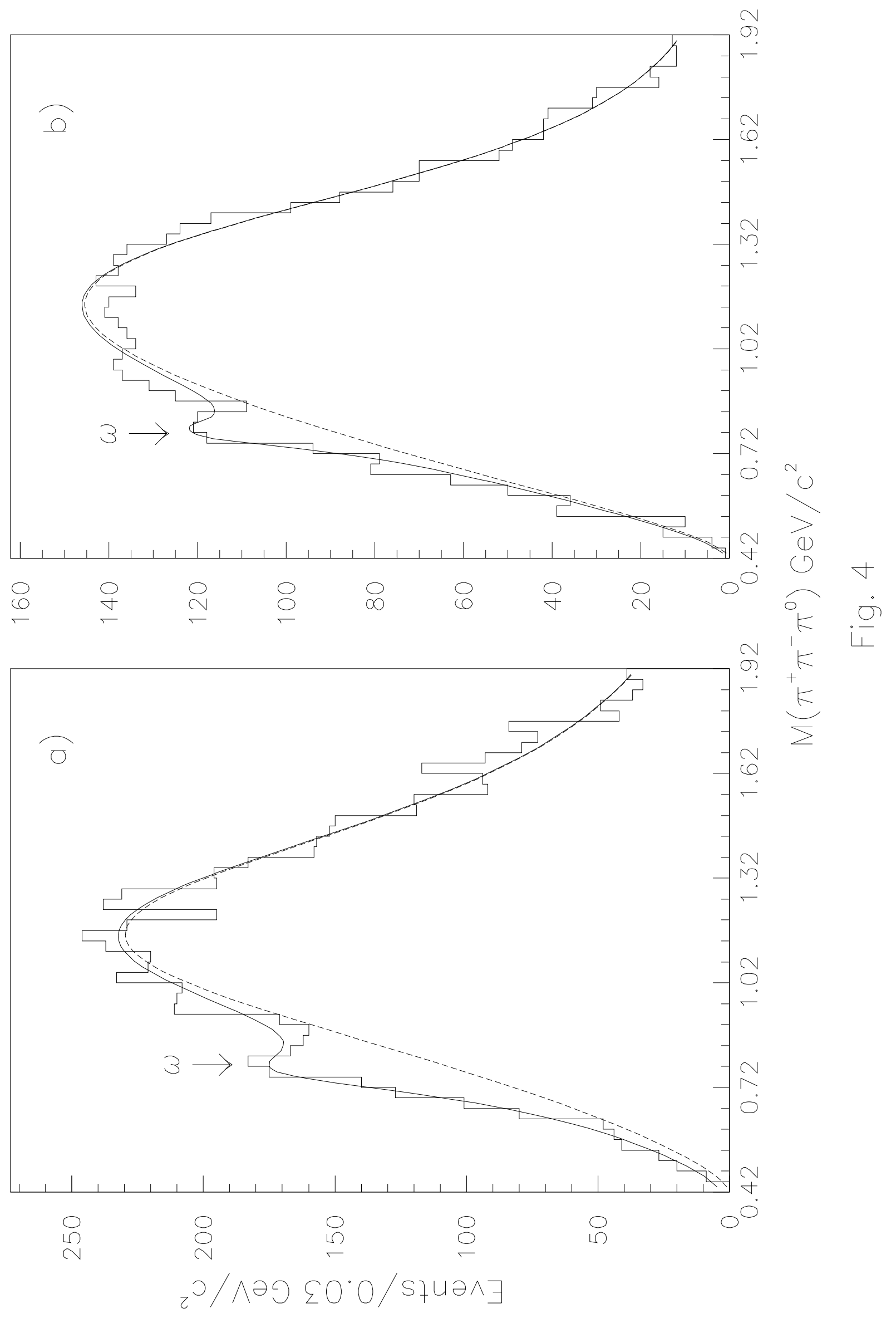


